(4) Symposium on Theoretical and Fundamental Physics

September 1953 at Kyoto (Japan) organized by the Japanese National Committee. Sections: Field Theory and Theory of Elementary Particles; Statistical Mechanics and Low Temperature Physics; Solid State Physics and Molecular Physics. Further information from Dr. Yoshra FujIokA, Secretary of the Conference, Science Council of Japan, Theno Park, Tokyo, Japan.

A. Perrier, Swiss National Committee of Physics

\title{
Instruments and Measurements Conference Stockholm 1952
}

Von der Königlichen Schwedischen Akademie der Ingenieurwissenschaften (IVA.) und der schwedischen Gesellschaft für technische Physik (TFF.) organisiert, fand vom 22. bis 25. Septemiber 1952 in Stockholm die 6. Konferenz über Instrumente und Messmethodik statt. Gleichzeitig wurde eine Ausstellung von Messinstrumenten durchgeführt (22. bis 30. September 1952).

Das Ziel dieser Tagungen ist die Darstellung und Diskussion neuer Messmethoden sowie die Vorführung neuer Instrumente zur Förderung der Forschung und der allgemeinen technischen Entwicklung. Die diesjährige Tagung wurde von rund 400 Teilnehmern besucht. In Parallelsitzungen wurden 80 Vorträge über Messtechnik auf folgenden Gebieten gehalten:

a) Mechanik, b) physikalische Chemie, c) Elektronik, d) Kernphysik, e) Spektroskopie, f) allgemeine Elektrik, g) industrielle Kontrolle.

Die Auszüge dieser Vorträge sind in den IVA. (Tidskrift för Teknisk-Vetenskaplig Forskning) Bd. 23, Heft 4 (1952), erschienen. Wie in den Vorjahren werden die vollständigen Referate in den "Transactions of Instruments and Measurements Conference" (Stockholm 1952) veröffentlicht werden.

Sehr interessant und reichhaltig war die Ausstellung von Instrumenten und insbesondere die Abteilung für Forschung. Hier hatten verschiedene Institute der skandinavischen Länder sowie die kernpliysikalischen Zentren von England und Frankreich in Bildern und Modellen einen Ausschnitt aus ihrer Tätigkeit gezeigt.

N. SCHAETII

\section{3. Österreichischer Mathematikerkongress vom 9. bis 14. September 1952 in Salzburg}

Die Österreichische Mathematische Gesellschaft gestaltet seit einiger Zeit ihre Jahresversammlung zu einem internationalen Mathematikertreffen aus, das auch dieses Jahr unter der Leitung des Vorsitzenden der Gesellschaft, Prof. Dr. H. INZINGER, aufs beste gelang, indem etwa 300 Delegierte aus den verschiedensten Ländern teilnahmen.

In der Sektion für angewandte Mathematik wurden folgende Vorträge gehalten: D. R. Rutherford (St. Andrews): The Relaxation Method. A. ZILLER (Strassburg): Valeur de $t_{n}$ du dernier terme du développement en série de Taylori. E. STIEFEL (Zürich): Uber die Methode der konjugierten Gradienten zur Auflosung von Systemen lineaver Gleichungen unter spezieller Berücksichtigung des Einsatzes von Rechenautomaten. S. EKELÖF (Göteborg) : Der mechanische Differentialanaly" sator der Chalmersschen Technischen Hochschule und einige damit gelöste Probleme. E. DORY (Löwen): Sur des ovientations récentes de l'enseignement supérieur dans 